\title{
Alterations in the Rheological Flow Profile in Conduit Femoral Artery during Rhythmic Thigh Muscle Contractions in Humans
}

\author{
Takuya OSADA ${ }^{*, \dagger}$ and Göran RÅDEGRAN ${ }^{\dagger, \ddagger}$ \\ *Department of Preventive Medicine and Public Health, Tokyo Medical University, Tokyo, 160-8402 Japan; \\ ${ }^{\dagger}$ The Copenhagen Muscle Research Centre, University of Copenhagen, Rigshospitalet, DK-2100 Copenhagen $\varnothing$, \\ Denmark; and ₹Department of Cardiothoracic Surgery, The Heart and Lung Division, Lund University Hospital, 22185 \\ Lund, Sweden
}

\begin{abstract}
The present study examined the rheological blood velocity profile in the conduit femoral artery during rhythmic muscle contractions at different muscle forces. Eight healthy volunteers performed one-legged, dynamic knee-extensor exercise at work rates of $5,10,20,30$, and $40 \mathrm{~W}$ at 60 contractions per minute. The time and space-averaged, amplitude-weighted mean $\left(V_{\text {mean }}\right)$ and maximum $\left(V_{\text {max }}\right)$ blood flow velocities in the common femoral artery were measured during the cardiosystolic phase (CSP) and cardiodiastolic phase (CDP) by the Doppler ultrasound technique. The $V_{\text {max }} / V_{\text {mean }}$ ratio was used as a flow profile index, in which a ratio of $\sim 1$ indicates a "flat velocity flow profile" and a ratio significantly $>1$ indicates a "parabolic velocity flow profile." At rest, the $V_{\text {max }} / V_{\text {mean }}$ ratio was $\sim 1.3$ and $\sim 1.8$ during the CSP and CDP, respectively. The $V_{\max } / V_{\text {mean }}$ ratio was higher $(p<0.01)$ during the CDP than during the CSP, both at rest and at all work rates. The $V_{\text {max }} / V_{\text {mean }}$ ratio during the CSP was higher $(p<0.01)$ at 30 and $40 \mathrm{~W}$ compared to at rest. The $V_{\text {max }} / V_{\text {mean }}$ ratio during the CDP was lower $(p$ $<0.05$ ) at 5 and $10 \mathrm{~W}$ compared to at rest. There was a positive linear correlation between blood flow and incremental work rates during both the CSP and CDP, respectively. Thus under resting
\end{abstract}

conditions, the findings indicate a "steeper" parabolic velocity profile during the CDP than during the CSP. The velocity profile during the CDP furthermore shifts to being less "steep" during rhythmic muscle contractions at lower intensities, but to being reelevated and normalized as at rest during higher intensities. The "steepness" of the parabolic velocity profile observed during the CSP at rest increased during muscle contraction at higher intensities. In conclusion, the blood velocity in the common femoral artery is parabolic both at rest and during exercise for both the CSP and CDP, indicating the persistence of laminar flow. The occurrence of any temporary slight disturbance or turbulence in the flow at the sight of measurement in the common femoral artery does consequently not induce a persisting "disturbed" and fully flat "plug-like" velocity profile. Instead, the "steepness" of the parabolic velocity profile is only slightly modified, whereby blood flow is not impaired. Thus the blood velocity profile, besides being influenced by the muscle contraction-relaxation induced mechanical "impedance," seems also to be modulated by the cardiac- and blood pressure-phases, consequently influencing the exercise blood flow response. [The Japanese Journal of Physiology 55: 19-28, 2005]

Key words: rheological blood flow profile, maximum and mean blood velocity, exercise hyperemia, rhythmic exercise, Doppler ultrasound.

During rhythmic muscle contractions, the blood velocity and its distribution of red blood cells moving in a conduit artery may be altered by the intramuscular pressure-induced mechanical hindrance to blood flow generated by the muscle contractions-relaxations and its temporal relation to the perfusion pressure, cardiac cycle, and state of vasodilatation [1-3]. Doppler ultrasound is furthermore a noninvasive technique that

Received on October 4, 2004; accepted on January 11, 2005; DOI: 10.2170/jjphysiol.R2074

Correspondence should be addressed to: Takuya Osada, Department of Preventive Medicine and Public Health, Tokyo Medical University, 6-1-1 Shinjuku, Shinjuku-ku, Tokyo, 160-8402 Japan. Phone: +81-3-5379-4339, Fax: +81-3-3226-5277, E-mail:

DENTACMAC@aol.com 
allows high temporal and spatial measurements of the blood velocity in conduit blood vessels. It is known that the blood velocity profile, representing the red blood cell velocity distribution in the conduit artery, varies between the cardiosystolic and cardiodiastolic phases at rest [4-7]. In the carotid and femoral artery at rest, it has previously been demonstrated that the blood velocity during the cardiosystolic phase has a less "steep" and somewhat "flatter" velocity profile while demonstrating a "steeper" parabolic velocity profile during the cardiodiastolic phase [4, 7-9]. In other words, at rest the maximum blood velocity $\left(V_{\max }\right.$; outer envelope velocity) is significantly higher than the mean blood velocity ( $V_{\text {mean }}$; time and spaceaveraged, and amplitude-weighted blood velocity) during the cardiodiastolic phase, whereas $V_{\max }$ is only slightly higher than $V_{\text {mean }}$ during the cardiosystolic phase. Therefore at rest there is a difference in the flow profile between the cardiosystolic and cardiodiastolic phases.

However, a lack of knowledge exists about the pattern of the blood velocity profile distribution in the conduit artery during rhythmic muscle exercise and whether there are variations specifically during the cardiosystolic and cardiodiastolic phases. Such alterations in the blood velocity profile are indeed of major importance to clarify because they may influence the blood flow response to exercise. Furthermore, even though limb femoral artery blood flow (FBF) is known to increase linearly with increasing work rate during one-legged, dynamic knee-extensor exercise (DKE) [1, 10-12], it is not clear whether the same is true during the cardiosystolic and cardiodiastolic phases, respectively

The hypothesis investigated in the present study was that the blood velocity profile and the absolute flow magnitude, besides being influenced by the mechanical hindrance of dynamic muscle contractions and different exercise intensities, also may be influenced by the cardiosystolic and cardiodiastolic phases, respectively. The present study therefore examined (i) the blood velocity profile in the common femoral artery expressed as $V_{\max }$ and $V_{\text {mean }}$ during the cardiosystolic and cardiodiastolic phases at rest and during DKE, and (ii) the magnitude of the hyperemic response determined from the blood velocity profile measurements. (iii) To what extent $V_{\max }$ may overestimate the calculation of blood flow compared with $V_{\text {mean }}$, traditionally used in Doppler ultrasound studies was also investigated.

\section{METHODS}

Subjects. Eight healthy male volunteers with a mean \pm SE (range) age of $26.6 \pm 1.4$ (22-36 years), height of $183 \pm 2(174-190) \mathrm{cm}$, and body weight of $77.3 \pm 2.9(64-90) \mathrm{kg}$ participated in the experiments. The subjects'participation varied from daily activities to regular endurance training. They had no previous history of cardiovascular disease, hypertension, or anemia. The subjects who volunteered were informed about the experimental procedures and the potential risks and discomfort, and they were told that they could withdraw at any time without consequences. They participated after providing signed informed consent. The experiments were carried out with approval from the Ethical Committees of Copenhagen and Fredriksberg (KF-01-013/96).

Experimental protocol. Before the experiments, all subjects were familiarized with the one-legged, dynamic knee-extensor exercise (DKE) model $[10,11]$. They trained at 60 contractions per minute (cpm) at work rates of $5,10,20,30$, and $40 \mathrm{~W}$ until they were comfortable and could fully relax the hamstring muscles. This ensured that the work was performed solely by the knee extensors of the quadriceps muscle group (Fig. 1). With the thigh horizontal, the lower leg moved up to a knee angle of $~ 150$ degrees; 180 degrees corresponds to the fully extended knee. The specific loads of $5,10,20,30$, and $40 \mathrm{~W}$ corresponded to applied weights of $0.083,0.167,0.333,0.5$, and $0.667 \mathrm{~kg}$, respectively. The work rate was calculated according to the dynamic knee-extensor ergometer model $[10,11]$, defined as work rate $(\mathrm{W})=($ contraction rate $[\mathrm{cpm}] / 60$ s) $\times($ distance of a knee-extensor revolution $[6 \mathrm{~m}]) \times$ (load weight $[\mathrm{kg}] \times 9.81\left[\mathrm{~m} / \mathrm{s}^{2}\right]$ ).

Peripheral arterial blood pressure (BP) was monitored continuously with a finger-cuff photoplethysmography device (Finapres ${ }^{\mathrm{TM}}$, Ohmeda 2300, Englewood, NJ) placed on the middle finger of the left hand. The finger cuff was maintained next to the inguinal ligament in a fixed position at the level of the femoral artery. Mean arterial BP was calculated by integrating the BP curve over time. The kicking muscle force was measured with a strain gauge (Fig. 1). The muscle force curve was used to represent the oscillations in intramuscular pressure because a good correlation between muscle force and intramuscular pressure within the knee extensor muscle group previously has been demonstrated during DKE $[1,2,13]$. The signals of all parameters were continuously recorded for approximately 4 min at steady-state DKE and stored in a computer using a MacLab data acquisition system 


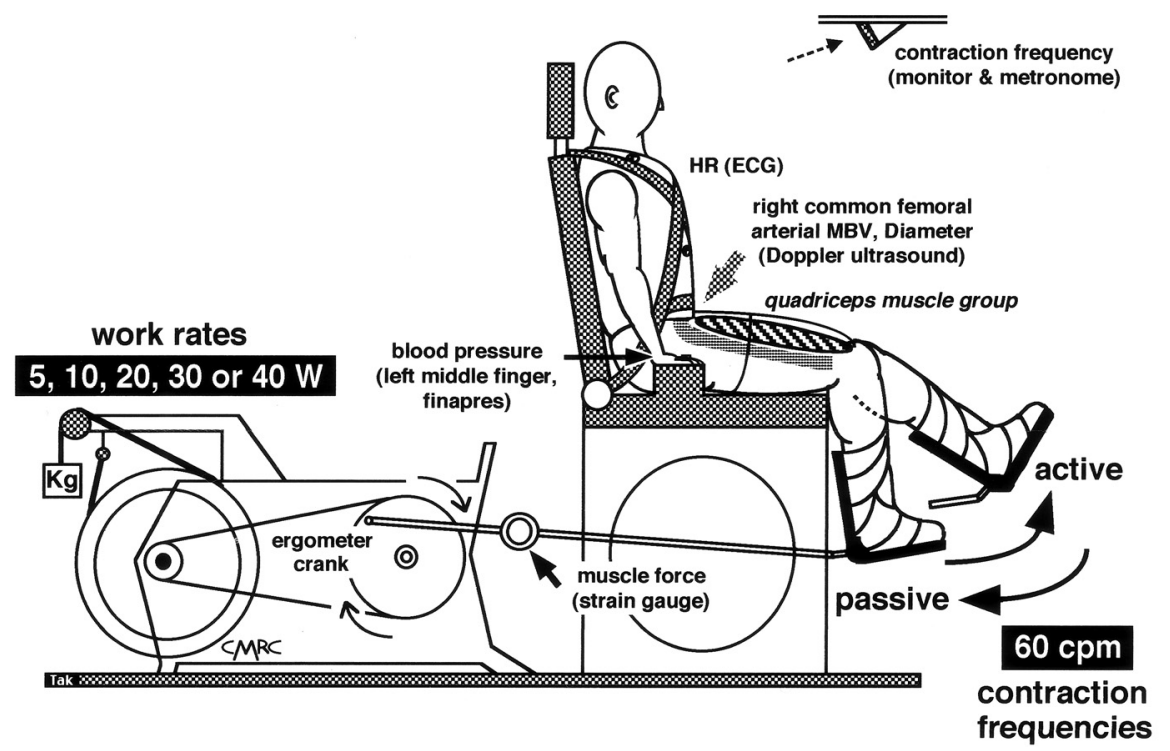

Fig. 1. One-legged, dynamic knee-extensor exercise (DKE) as a rhythmic muscle contraction model. The volunteers performed a one-legged, dynamic knee-extensor exercise (DKE), i.e., rhythmic thigh muscle contractions at incremental work rates of 60 contractions per minute (cpm). Continuous steady-state measurements were performed at rest and during DKE.

(Chart v.3.5.7 software, ADInstruments, Sydney, Australia). The experimental protocols were performed on the same day. It was verified that a sufficient amount of time between the knee-extensor exercise bouts was allowed for blood flow to return to control resting levels. Belts attached to the seat fixed the upper body and both thighs. The FBF measurements were performed by Doppler ultrasound at steady-state after $3 \mathrm{~min}$ of DKE $[2,3]$, as previously described [1]. The contraction rhythm was maintained by following the pace of a visible and audible metronome and by watching the contraction rate displayed in real time on a monitor.

Blood velocity measurements with Doppler ultrasound. Doppler ultrasound is a noninvasive technique that provides high temporal and spatial measurements of the blood velocity, representing the red blood cell velocity distribution in conduit blood vessels. The source of the signal producing the Doppler shift frequency is the "red blood cells." The blood velocity measured by Doppler ultrasound thus reflects the hemodynamic characteristics of the red blood cells [8].

The procedures for blood velocity and flow measurements in the femoral artery have previously been validated and been shown to produce accurate absolute values at rest and during DKE [1]. The equipment used was a Doppler ultrasound (model CFM 800, Vingmed Sound, Horten, Norway) equipped with an annular phased array transducer (Vingmed Sound) probe (11.5 $\mathrm{mm}$ diameter) operating at an imaging frequency of $7.5 \mathrm{MHz}$ and variable Doppler frequencies of 4.0-6.0 MHz (high-pulsed repetition frequency mode, 4-36 kHz). The time and space-averaged, and amplitude-weighted mean blood velocity and maximum blood velocity (maximum outer envelope or peak blood velocity) were defined as $V_{\text {mean }}[1,12$, 14] and $V_{\max }$ [15-19], respectively. The $V_{\max }$ obtained in the present study was expressed as the blood velocity measured at the center of the vessel. Each blood velocity parameter was measured in relation to the blood pressure curve.

Location of measured vessel. The site for vessel diameter determination and blood velocity measurements in the common femoral artery was distal to the inguinal ligament, $\sim 1-2 \mathrm{~cm}$ above the bifurcation into the superficial and profunda femoral branch [3, 18]. This position was chosen to minimize any turbulence from the bifurcation and influence of blood flow from the inguinal region. Furthermore, the arterial diameter is unaffected by the contractions and relaxations at this site proximal to the muscle location. The blood velocity measurements were performed with the probe at an insonation angle as low as physically possible and always below 60 degrees [20]. The mean value of the probe angle in the present study was $\sim 45.5$ \pm 0.8 degrees and remained constant throughout experiments for the individual subjects. Special care was taken to ensure that the insonation angle did not vary and that the sample volume was precisely positioned in the center of the vessel and adjusted to cover the width of the diameter. In the present study, the average width of the circular sampling volume was $11 \mathrm{~mm}$. The Doppler system measured the blood velocity profile within this short band of sampling volume. With regards to the laminar, nonpulsatile steady flow of a Newtonian fluid in a straight rigid cylindrical tube, it is known 


\section{T. OSADA and G. RÅDEGRAN}

that the flat velocity profile at a proximal site near a tube entrance progressively evolves to a parabolic velocity profile downstream at a distal site as a result of alterations in the boundary layer $[8,21-23]$. In the present study, no major concern of any downstream flow profile alteration from the inlet was accounted for in our investigation of a non-Newtonian fluid [24] and a pulsatile arterial blood velocity profile.

Calculation of blood flow. The heart rate and the systolic and diastolic diameters of the femoral artery were measured on a monitor in relation to the ECG. The mean vessel diameter was calculated in relation to the temporal duration of the blood pressure curve: ([systolic diameter value $\times 1 / 3]+[$ diastolic diameter value $\times 2 / 3]$ ) [1]. The diameter measurements were obtained under perpendicular insonation. Signal interference by blood turbulence near the vascular wall was reduced with a low-velocity rejection filter. FBF was calculated by multiplying the cross-sectional area (area $\left.=\pi \times[\text { diameter } / 2]^{2}\right)(\mathrm{CSA})$ of the femoral artery with the angle corrected, time and space averaged, and amplitude-signal intensity weighted blood velocity $\left(V_{\text {mean }}\right.$ or $\left.V_{\text {max }}\right)$ where $\mathrm{FBF}_{\text {mean }}=V_{\text {mean }} \times \mathrm{CSA}$ $\times 6 \times 10^{4}(\mathrm{l} / \mathrm{min})$ and $\mathrm{FBF}_{\text {max }}=V_{\text {max }} \times \mathrm{CSA} \times 6 \times 10^{4}$ $(l / \mathrm{min})$. The constant $6 \times 10^{4}$ is the conversion factor from $\mathrm{m} / \mathrm{s}$ to $l / \mathrm{min}$ [1]. Because the femoral arterial diameter has been found not to change from rest to $\mathrm{DKE}$, the diameter measurements at rest were used for the calculation of FBF during DKE $[1,12,18]$.

The $V_{\text {max }} / V_{\text {mean }}$ ratio during the cardiosystolic and cardiodiastolic phases. The velocity profile index was expressed as the $V_{\text {max }} / V_{\text {mean }}$ ratio [25]. The cardiosystolic phase started at the upstroke before minimum BP and ended before the second dicrotic BP notch. The cardiodiastolic phase was defined between the notch before the second dicrotic BP notch and the minimum BP before the upstroke (Fig. 2). The velocity profile $\left(V_{\max } / V_{\text {mean }}\right.$ ratio) index was determined for the cardiosystolic and cardiodiastolic phases. Changes in the velocity profile were estimated from changes in the $V_{\max } / V_{\text {mean }}$ ratio. For a parabolic-like velocity profile, the $V_{\max } / V_{\text {mean }}$ ratio is significantly $>1.0$. The greater increase in $V_{\max }$ compared to $V_{\text {mean }}$ implies a steeper parabolic-like profile. For a flat pluglike velocity profile, the $V_{\max } / V_{\text {mean }}$ ratio is $\sim 1.0$ [25]. The velocity profile during muscle contraction was compared with the flow profile at rest during both the cardiosystolic and cardiodiastolic phases.

Intraobserver variability. Before the experiments, extensive operator training was performed to minimize any intraobserver variability with the Dop- pler ultrasound technique during rest and DKE. At rest, the coefficients of variation determined from repeated blood velocity measurements were $3.4 \pm 0.3 \%$.
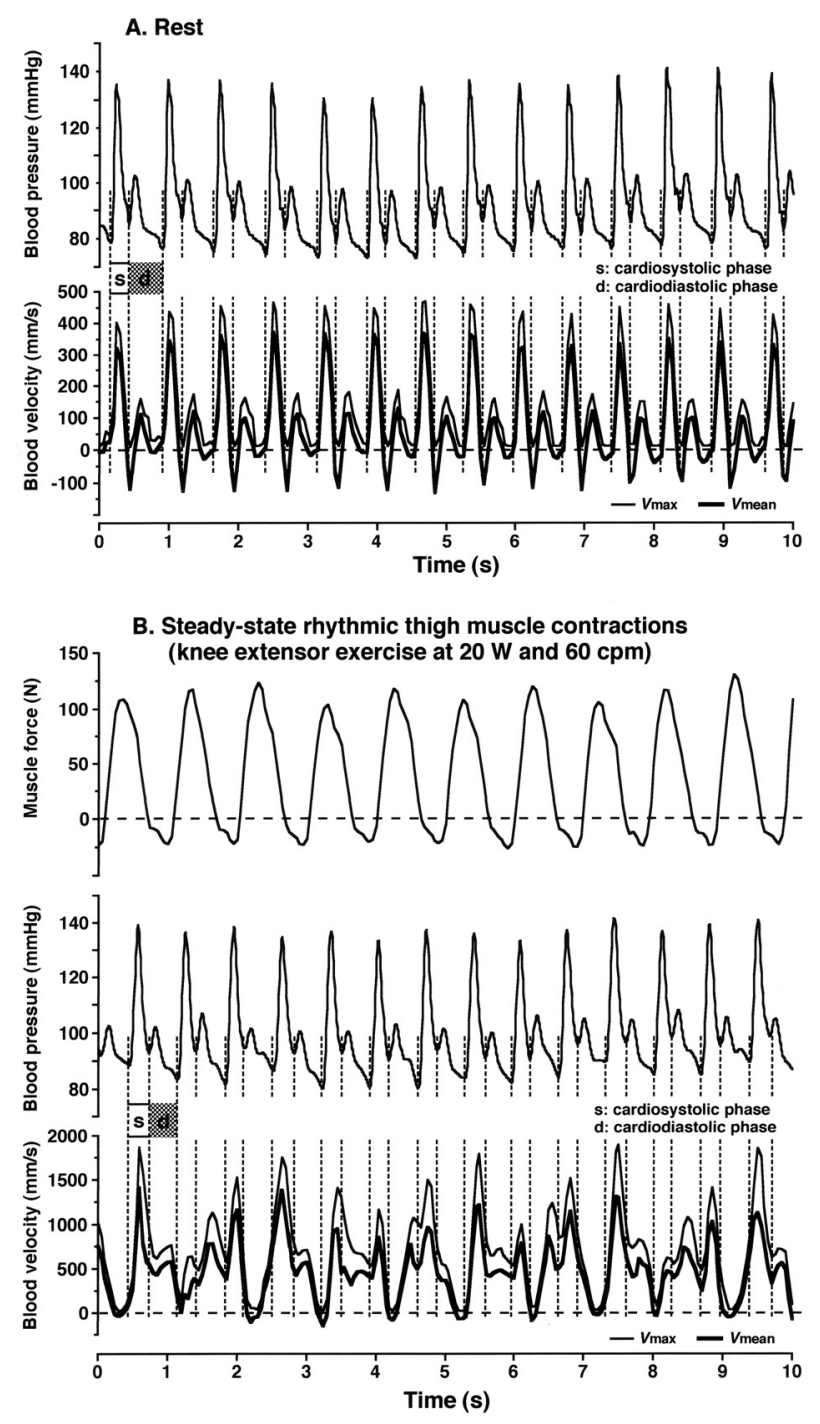

Fig. 2. Analysis of $\boldsymbol{V}_{\max }$ and $\boldsymbol{V}_{\text {mean }}$ during the cardiosystolic and cardiodiastolic phases at rest and during exercise. A: $V_{\text {max }}, V_{\text {mean }}$, and blood pressure at rest; B: $V_{\text {max }}$, $V_{\text {mean }}$, muscle force, and blood pressure during steady-state DKE at $20 \mathrm{~W}$ and 60 contractions per minute in one subject. $V_{\max }$ and $V_{\text {mean }}$ during the cardiosystolic and cardiodiastolic phases were measured in parallel with the blood pressure curve. The blood velocity fluctuated in relation to the state of vasodilation, the muscle contraction-relaxation cycle (indicated by the oscillations in muscle force), and the variation in the blood pressure curve. The cardiosystolic phase (s) and the cardiodiastolic phase (d) are shown. The thin line indicates $V_{\text {max }}$, and the thick line indicates $V_{\text {mean }}$. The $V_{\max }$ and $V_{\text {mean }}$ stated in Table 1 were determined as the "average" transient maximum (outer envelope) and mean blood velocity values (the amplitude-weighted time-andspatial average), respectively, for the cardiosystolic and cardiodiastolic phases. 
Limb Flow Profile during Muscle Contractions

Table 1. Average $V_{\max }$ and $V_{\text {mean }}$ at rest and during exercise for the cardiosystolic and cardiodiastolic phases.

\begin{tabular}{|c|c|c|c|c|c|}
\hline & \multicolumn{2}{|c|}{ Cardiosystolic phase } & \multicolumn{2}{|c|}{ Cardiodiastolic phase } & \multirow{2}{*}{$\begin{array}{l}\text { Sampling numbers of cardiac cycle } \\
\text { for measurements }\end{array}$} \\
\hline & $V_{\text {mean }}(\mathrm{mm} / \mathrm{s})$ & $V_{\max }(\mathrm{mm} / \mathrm{s})$ & $V_{\text {mean }}(\mathrm{mm} / \mathrm{s})$ & $V_{\max }(\mathrm{mm} / \mathrm{s})$ & \\
\hline Rest & $162 \pm 17$ & $213 \pm 26^{*}$ & $36 \pm 3$ & $63 \pm 5^{\star}$ & $60 \pm 12$ \\
\hline $5 \mathrm{~W}$ & $590 \pm 60^{\#}$ & $798 \pm 69^{*} \#$ & $248 \pm 30^{\#}$ & $362 \pm 32^{\star \#}$ & $184 \pm 33$ \\
\hline $10 \mathrm{~W}$ & $711 \pm 71^{\# \dagger}$ & $953 \pm 75^{* \#+}$ & $314 \pm 28^{\# \dagger}$ & $465 \pm 26^{*}+$ & $164 \pm 23$ \\
\hline $20 \mathrm{~W}$ & $838 \pm 68^{\# \dagger}$ & $1,142 \pm 75^{\text {\#\# }}$ & $416 \pm 31^{\# \dagger}$ & $620 \pm 33^{* \#+}$ & $183 \pm 18$ \\
\hline $30 \mathrm{~W}$ & $946 \pm 77^{\# \dagger}$ & $1,364 \pm 91^{* \#+}$ & $510 \pm 33^{\#+}$ & $805 \pm 38^{* \#+}$ & $137 \pm 15$ \\
\hline $40 \mathrm{~W}$ & $979 \pm 89^{\# \dagger}$ & $1,469 \pm 115^{\star \# \dagger}$ & $592 \pm 32^{\# \dagger}$ & $926 \pm 43^{* \#+}$ & $102 \pm 16$ \\
\hline
\end{tabular}

The average maximum $\left(V_{\max }\right)$ and mean $\left(V_{\text {mean }}\right)$ blood velocity increased $(p<0.05)$ for each consecutive exercise intensity when compared to $5 \mathrm{~W}\left(^{\dagger}\right)$ and at rest $\left({ }^{\#}\right) . V_{\max }$ was larger $(p<0.05)$ than $V_{\text {mean }}$ at each condition $\left({ }^{*}\right)$ for both the cardiosystolic and cardiodiastolic phases. The sampling number of measurements was the same between the

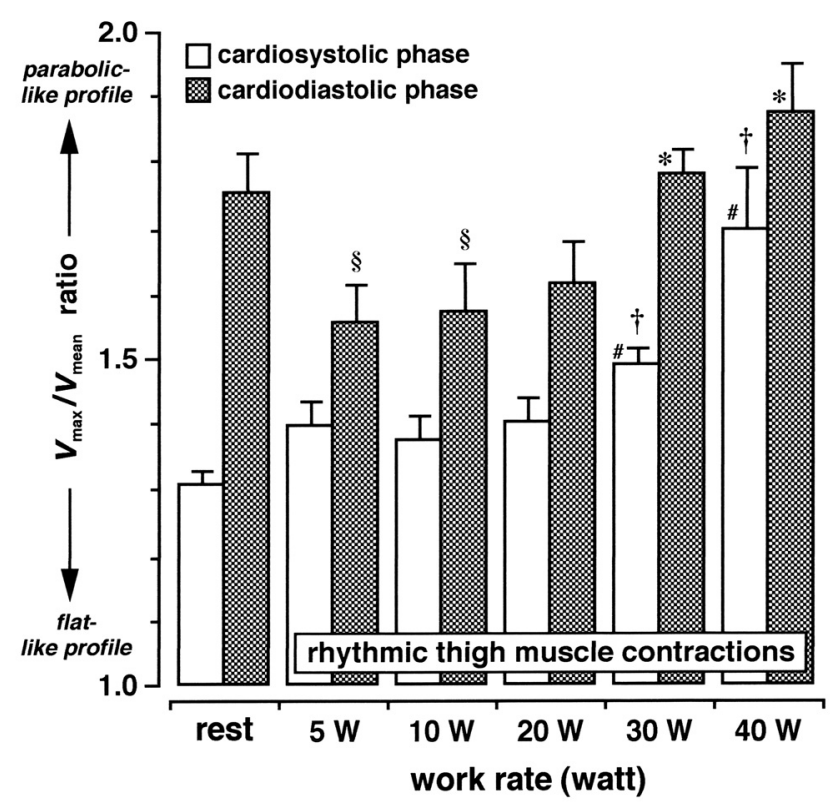

Fig. 3. The $V_{\max } / V_{\text {mean }}$ ratio during the cardiosystolic and cardiodiastolic phases at rest and during exercise. A significantly $(p<0.01)$ higher $V_{\text {max }} / V_{\text {mean }}$ ratio was found during the cardiodiastolic phase compared to the cardiosystolic phase at rest and for each work rate during rhythmic muscle contractions (not indicated in the figure). The $V_{\text {max }} / V_{\text {mean }}$ ratios during the cardiosystolic phase at 30 and $40 \mathrm{~W}$ were higher $\left({ }^{\dagger} p<0.01\right)$ than at rest. The $V_{\text {max }} / V_{\text {mean }}$ ratios during the cardiodiastolic phase at 5 and $10 \mathrm{~W}$ were lower $(\$ p<0.05)$ than at rest. The $V_{\text {max }} / V_{\text {mean }}$ ratios during the cardiosystolic and cardiodiastolic phases were significantly higher ( ${ }^{*} \#<0.05$ ) at $30 \mathrm{~W}$ and $40 \mathrm{~W}$ than at $5 \mathrm{~W}$. The values are expressed as means \pm SE.

These values represent the minimal level of quality control of the method at rest [3, 26, 27]. During exercise, the coefficients of variation for the repeated cardiosystolic and cardiodiastolic phases (cardiac cycle) at each work rate. The number of samplings was sufficient for evaluating the magnitude of average blood velocity. Sig-

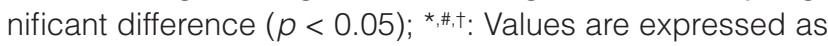
means \pm SE.

blood velocity measurements were $<5 \%$ [3], which is in agreement with the oscillations in blood flow because of variations in the intramuscular pressure and its relation to the cardiac and blood pressure phases.

Statistical analysis. Parametric statistics was used for data analysis (i.e., multiple analysis of variance for repeated measures and Scheffés significant difference post hoc tests when we compared more than two groups over time; a paired $t$-test when we compared two groups). A $p$ value $<0.05$ was considered statistically significant. A $p=\mathrm{NS}$ indicates not statistically significant. The values are mean \pm SE.

\section{RESULTS}

The femoral arterial diameter at rest was $10.9 \pm 0.3$ $\mathrm{mm}$. The $V_{\max }$ and $V_{\text {mean }}$ as well as the $V_{\text {max }} / V_{\text {mean }}$ ratios during the cardiosystolic and cardiodiastolic phases at rest and during DKE are shown in Table 1 and Fig. 3 . The $V_{\max } / V_{\text {mean }}$ ratio during the cardiosystolic phase was significantly higher $(p<0.01)$ at 30 and $40 \mathrm{~W}$ than at rest. The $V_{\text {max }} / V_{\text {mean }}$ ratio during the cardiodiastolic phase was significantly lower $(p<0.05)$ at 5 and 10 $\mathrm{W}$ than when at rest. Moreover, the $V_{\text {max }} / V_{\text {mean }}$ ratio was lower $(p<0.01)$ during the cardiosystolic phase than during the cardiodiastolic phase both at rest and during DKE (Fig. 3). There was a positive linear correlation $(p<0.0001)$ between $V_{\text {max }}$ and $V_{\text {mean }}$ during the cardiosystolic and cardiodiastolic phases (Fig. 4). The regression lines estimate $V_{\max }$ for a given $V_{\operatorname{mean}} ; V_{\max }=$ $8.916+1.431 \times V_{\text {mean }}$. Thus $V_{\max }$ is $\sim 1.43$-fold higher than $V_{\text {mean }}$. Therefore the $V_{\text {mean }}$ during DKE may be estimated to be $\sim 70 \%$ of $V_{\max }$. 


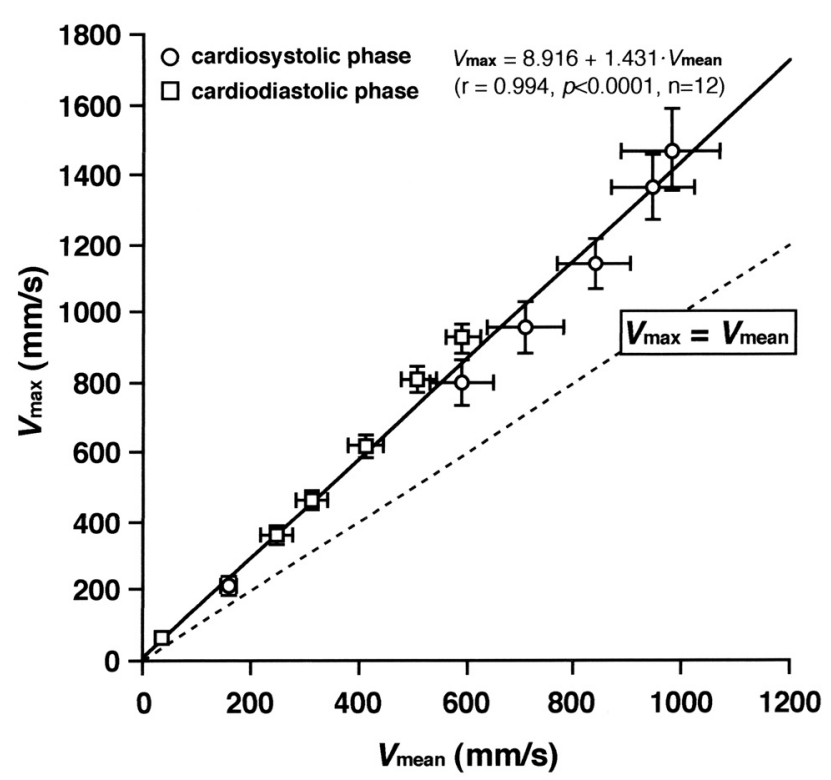

Fig. 4. The relationship between $\boldsymbol{V}_{\text {max }}$ and $\boldsymbol{V}_{\text {mean }}$ during the cardiosystolic and cardiodiastolic phases at rest and during exercise. There was a positive linear correlation between $V_{\text {max }}$ and $V_{\text {mean }}$ during both the cardiosystolic (circles) and cardiodiastolic phases (squares). The dotted line shows the line of identity $\left(V_{\max }=V_{\text {mean }}\right)$. The values are expressed as means \pm SE.

The $\mathrm{FBF}_{\text {max }}$ and $\mathrm{FBF}_{\text {mean }}$ during the cardiosystolic and cardiodiastolic phases at rest and during DKE are shown in Fig. 5. There was a positive linear correlation during $\mathrm{DKE}$ between $\mathrm{FBF}_{\text {max }}$ and $\mathrm{FBF}_{\text {mean }}$ versus the incremental work rates, respectively, during the cardiosystolic $\left(p<0.001, r=0.991\right.$ for $V_{\max }$ during the cardiosystolic phase; $p<0.01, r=0.967$ for $V_{\text {mean }}$ during the cardiosystolic phase) and cardiodiastolic phases $\left(p<0.001, r=0.997\right.$ for $V_{\max }$ during the cardiodiastolic phase, and $p<0.001, r=0.998$ for $V_{\text {mean }}$ during the cardiodiastolic phase). The regression lines during the cardiosystolic and cardiodiastolic phases reflect the FBF value at a given work rate (WR) and phase; i.e., $\mathrm{FBF}_{\text {max-systole }}(l / \mathrm{min})=4.147+0.110 \times \mathrm{WR}$; $\mathrm{FBF}_{\text {mean-systole }}=3.240+0.063 \times \mathrm{WR} ; \mathrm{FBF}_{\text {max-diastole }}=$ $1.623+0.093 \times \mathrm{WR} ; \mathrm{FBF}_{\text {mean-diastole }}=1.146+0.056 \times$ WR. During DKE at the cardiosystolic phase, $\mathrm{FBF}_{\text {max }}$ was higher $(p<0.05)$ than $\mathrm{FBF}_{\text {mean }}$ at all work rates. During DKE at the cardiodiastolic phase, $\mathrm{FBF}_{\text {max }}$ was higher than $\mathrm{FBF}_{\text {mean }}$ at 30 and $40 \mathrm{~W}(p<0.05)$. Both $\mathrm{FBF}_{\text {max }}$ and $\mathrm{FBF}_{\text {mean }}$ were significantly higher $(p<$ $0.01)$, respectively, during the cardiosystolic than during the cardiodiastolic phase at all work rates.

The increase in the $\mathrm{FBF}_{\text {exercise }} / \mathrm{FBF}_{\text {rest }}$ ratio was greater $(p<0.05)$ during the cardiodiastolic phase than during the cardiosystolic phase for both the mean and maximum values. A significant increase in the mean and maximum FBF values during DKE was

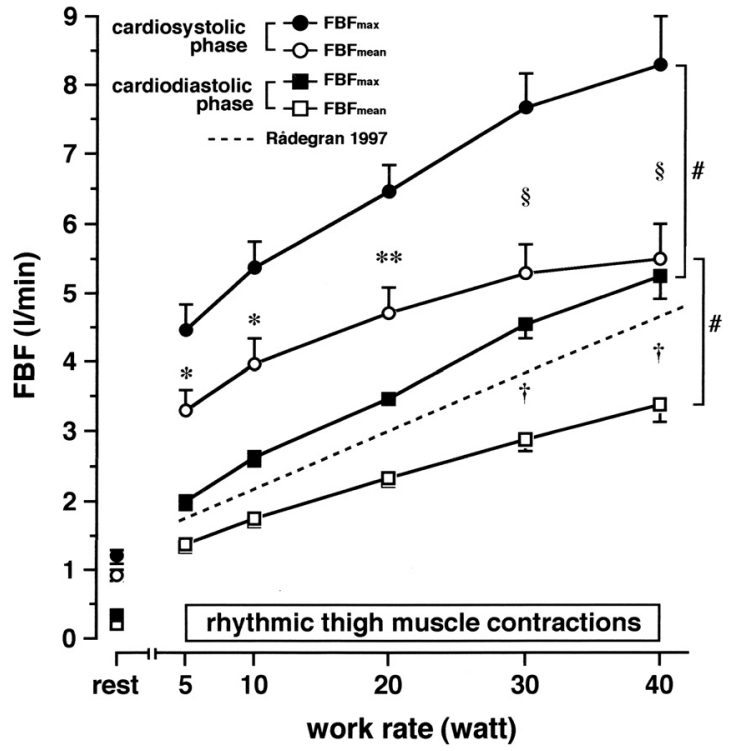

Fig. 5. $\mathrm{FBF}_{\text {max }}$ and $\mathrm{FBF}_{\text {mean }}$ during the cardiosystolic and cardiodiastolic phases at rest and during exercise. There was a positive linear correlation between FBF (max [solid circles] and mean [open cirlces] in the cardiosystolic phase, as well as max [solid squares] and mean [open squares] in the cardiodiastolic phase) and work rate during rhythmic thigh muscle contractions. The dotted line between the cardiosystolic FBF $_{\text {mean }}$ (open circles) and cardiodiastolic $\mathrm{FBF}_{\text {mean }}$ (open squares) at each work rate indicates the regression line $\left(\mathrm{FBF}_{\text {mean }}=1.317+0.084 \times \mathrm{WR}\right)$ previously reported by Rådegran [1]. The significant difference between $\mathrm{FBF}_{\text {max }}$ and $\mathrm{FBF}_{\text {mean }}$ during the cardiosystolic $\left({ }^{*} p<\right.$ $\left.0.05,{ }^{* *} p<0.01,{ }^{\S} p<0.001\right)$ and cardiodiastolic phases $\left({ }^{\dagger} p\right.$ $<0.05)$ indicate that. FBF was higher for the cardiosystolic than for the cardiodiastolic phases at all work rates $\left({ }^{\#} p<\right.$ 0.05). The values are expressed as means \pm SE.

seen only during the cardiodiastolic phase (Fig. 6). The heart rate was significantly higher $(p<0.05)$ at 30 and $40 \mathrm{~W}$ compared to $5 \mathrm{~W}$ and at rest. Although the duration of the cardiosystolic phase was similar $(p=\mathrm{NS})$ at rest and at all work rates, the duration of the cardiodiastolic phase was significantly shorter $(p<$ $0.05)$ at 30 and $40 \mathrm{~W}$ than at $5 \mathrm{~W}$ and rest, respectively (Fig. 7).

\section{DISCUSSION}

The present study described the features of blood flow distribution expressed as a blood velocity profile in the conduit femoral artery in humans. This was measured during rhythmic muscle contractions of the thigh muscle (DKE) at various work rates. The major finding in the present study was an altered blood velocity profile in response to rhythmic muscle contractions at differ- 


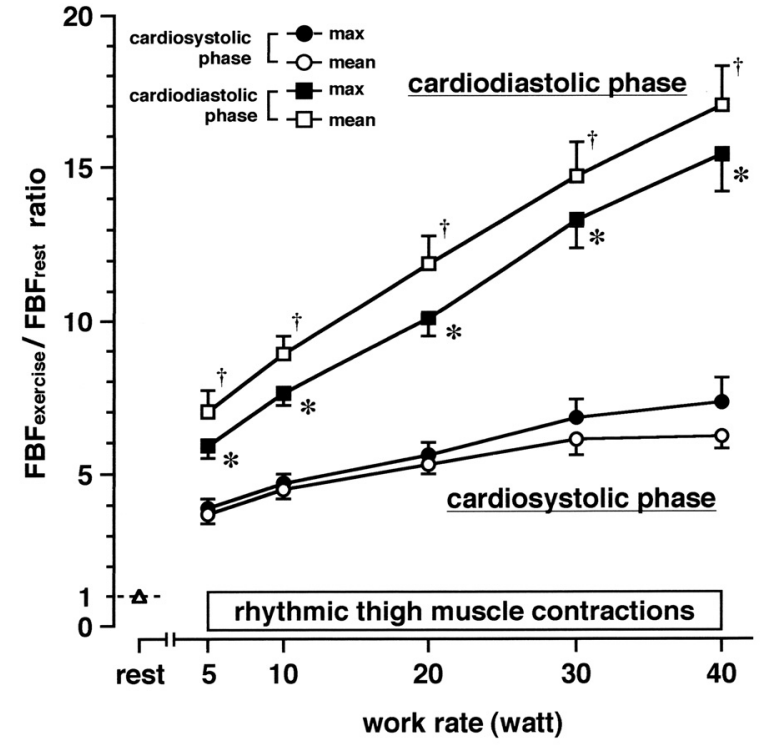

Fig. 6. $\mathrm{FBF}_{\text {exercise }} / \mathrm{FBF}_{\text {rest }}$ ratio during the cardiosystolic and cardiodiastolic phases. The increase in the $\mathrm{FBF}_{\text {exercise }}$ $\mathrm{FBF}_{\text {rest }}$ ratio was significantly higher $(p<0.05)$ during the cardiodiastolic phase than during the cardiosystolic phase for both $\mathrm{FBF}_{\text {mean }}$ and $\mathrm{FBF}_{\text {max }}$, respectively. The $\mathrm{FBF}_{\text {exercise }}$ / $\mathrm{FBF}_{\text {rest }}$ ratio was higher for the cardiodiastolic than for the cardiosystolic phase for both $\mathrm{FBF}_{\text {mean }}\left({ }^{\dagger} p<0.05\right)$ and $\mathrm{FBF}_{\text {max }}$ $\left({ }^{*} p<0.05\right)$. The values are expressed as means \pm SE.

ent exercise intensities. The blood velocity profile was assessed by the use of $V_{\max }$ and $V_{\text {mean }}$. The relationship between $V_{\text {max }}$ and $V_{\text {mean }}$ was evaluated not only at rest, but also during exercise. The following discussion covers the methodological implications for assessing the exercising blood velocity profile and variations in the hyperemic response between the cardiosystolic and cardiodiastolic phases during rhythmic muscle contractions.

Blood velocity profile at rest. At rest, the $V_{\max } /$ $V_{\text {mean }}$ ratios were higher at the cardiodiastolic phase than at the cardiosystolic phase (Fig. 3). This suggests that the blood velocity profile is "steeper" paraboliclike during the cardiodiastolic phase than during the cardiosystolic phase (The profile tends to be flatter.). This is in agreement with previous findings showing a relatively higher blood velocity in the center of the vessel and lower velocities near the vascular walls during the cardiodiastolic phase and a similar velocity between the center and the near wall during the cardiosystolic phase $[5,7,8,21,25]$. The Doppler spectral signal in human conduit vessels such as the carotid and femoral artery may represent $V_{\text {max }}$ by "a straight spectral line (narrow band) or a flat velocity profile" during the resting cardiosystolic phase, suggesting slightly more "uniformity in the blood velocities in a near-blunted pattern." Conversely, the spectrum line

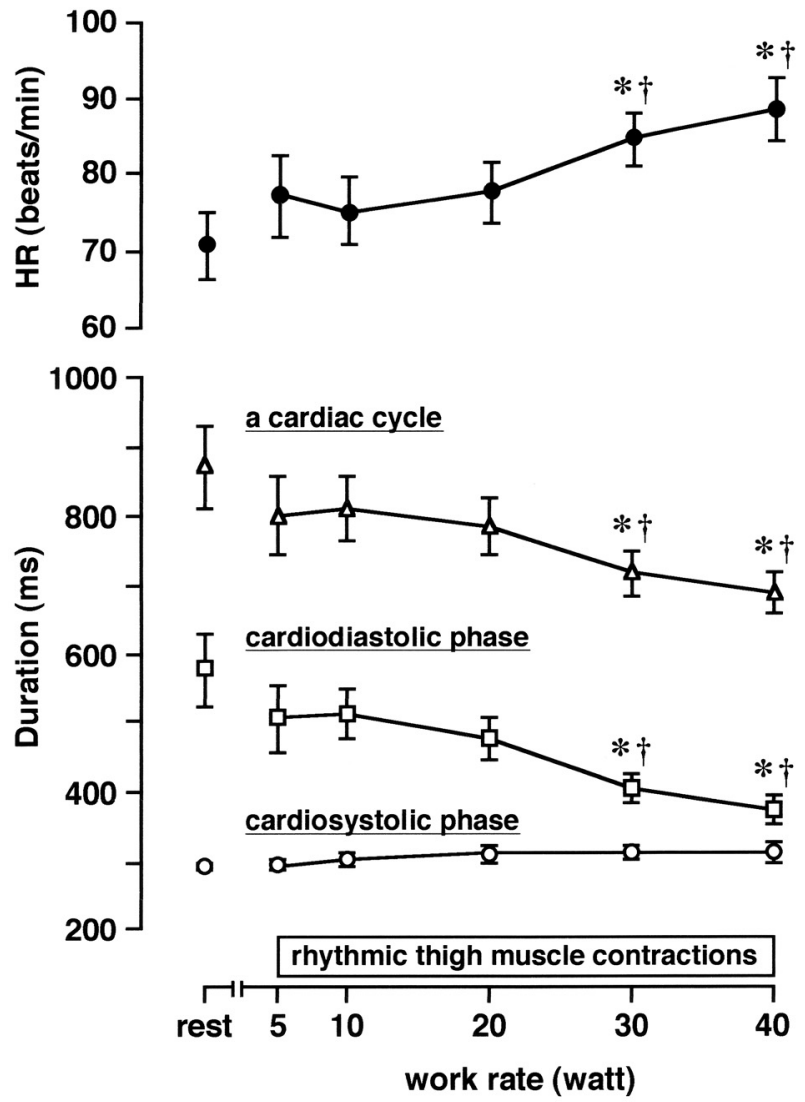

Fig. 7. Heart rate and duration of the cardiac cycle and the cardiosystolic and cardiodiastolic phases. Heart rate was significantly higher $(p<0.05)$ at 30 and $40 \mathrm{~W}$ than at $5 \mathrm{~W}$ and at rest. Although the duration of the cardiosystolic phase was similar ( $p=N S$ ) at rest and during each of the incremental work rates, the duration of the cardiodiastolic phase was significantly shorter $(p<0.05)$ at 30 and $40 \mathrm{~W}$ compared to $5 \mathrm{~W}$ and at rest. ( ${ }^{*} p<0.05 \mathrm{vs}$. at rest and ${ }^{\dagger} p<$ 0.05 vs. at $5 \mathrm{~W}$ ) The values are expressed as means \pm SE.

broadens (wide band) during the cardiodiastolic phase, suggesting that "the blood cells move with more different velocities in a parabolic-like pattern" similar to laminar flow with relatively lower blood acceleration [8]. In other words, the dispersion of blood cells in the vessel is different between the cardiosystolic and the cardiodiastolic phases because of the combination of steady and pulsatile flow in the artery $[5,7,25]$. The difference in the velocity profile between the cardiosystolic and the cardiodiastolic phases at rest results from a variety of factors such as flow acceleration, vessel geometry, and blood viscosity [8].

Rhythmic muscle contraction induced alterations in blood velocity profile. The major findings in the present study were that the index-ratio used to describe the blood velocity profile varied at each work rate during the cardiosystolic and cardiodiastolic phases. At lower muscle contraction forces, the 
$V_{\text {max }} / V_{\text {mean }}$ ratio during the cardiodiastolic phase temporarily shifted toward a less "steep" parabolic velocity profile, which was sustained during the cardiosystolic phase. However, at higher muscle contraction forces the cardiosystolic phase $V_{\text {max }} / V_{\text {mean }}$ ratio shifted toward a "steeper" parabolic-like velocity profile, which was also maintained during the cardiodiastolic phase.

The mechanisms of alteration in the blood velocity profile at the different muscle contraction intensities are still unknown. The rhythmic muscle contractions may possibly influence the blood velocity profile through various factors such as alterations in the intramuscular pressure and the state of vasodilatation and its relation to the perfusion pressure, which was not determined under resting conditions. It may be speculated that the "steeper" parabolic-like blood velocity profile at higher intensities $(30-40 \mathrm{~W})$ during the cardiosystolic and cardiodiastolic phases (Fig. 3) may be due to the elevated oscillations of intramuscular pressure, which may impede blood flow and influence the intravascular pressure upstream in the vascular bed. This is in contrast to the more uniform, blunted, and less "steep" parabolic blood velocity profile, which may result from lower intramuscular pressure and lower mechanical hindrance at work rates below 20 W. It could also be that the lower muscle contraction forces along with the lower intravascular pressure may accelerate the perfusion with all blood moving at similar speed. It may be suggested that the superimposed muscle contraction bouts may further influence the blood velocity profile, depending on whether the vessels supplying the vascular bed temporarily are vasodilated or vasoconstricted. Additionally, the blood velocity profile during muscle contractions are further influenced by the blood viscosity, which varies with shear rate as well as the inertial effect of the blood fluid as it accelerates and decelerates during cardiac pumping [8]. In general, higher blood velocities such as in the aorta may induce turbulent flow with larger blood cell vortexes occurring above the critical Reynolds number [23, 28, 29]. Therefore it is expected that alterations in the blood velocity profile as a result of muscle contraction-induced hyperemia (higher blood velocity) should be different than under resting conditions.

It may furthermore be speculated that the duration of the cardiosystolic and cardiodiastolic phases, which vary according to the heart rate, may influence the blood velocity profile. In the present study, however, the duration of the cardiodiastolic phase at higher intensities (increased heart rate) was significantly re- duced compared to $5 \mathrm{~W}$ and at rest, despite maintaining the cardiosystolic phase interval (Fig. 7).

Relationship between $V_{\text {max }}$ and $V_{\text {mean }}$ at rest and during rhythmic muscle contractions. $V$ $[16-19,30]$ and $V_{\text {mean }}[1,12,14,15,30]$ have both been used to calculate blood flow at rest and during various types of exercise. To what extent $V_{\max }$ may overestimate the calculation of FBF compared with using $V_{\text {mean }}$ at rest and during DKE was also addressed. In several previous studies, the $V_{\text {max }}$ have been determined by manually tracing the maximum envelope or by using an automated tracing system of the blood velocity. Measuring "exercising" blood velocity does present some challenges. Artifacts produced by a slight movement of the sampling volume during bouts of muscle contraction may make it difficult to determine $V_{\text {mean }}$ even if the maximum envelope $\left(V_{\max }\right)$ is clearly defined. In other instances, the instrument may have a system for determining $V_{\max }$ but none for measuring $V_{\text {mean }}$. Measuring the maximum envelope of blood flow and the estimating of $V_{\text {mean }}$ from $V_{\text {max }}$ may therefore be a complementary option. Because $V_{\max }$ was found to be $\sim 1.43$-fold higher than $V_{\text {mean }}$ (Fig. 4), FBF may be overestimated by $\sim 43 \%$ when using $V_{\max }$ for the blood flow calculation, assuming a constant vessel diameter value. Therefore in the present study, $V_{\text {mean }}$ was estimated to be $\sim 70 \%$ of $V_{\max }$ during rhythmic DKE. These findings were in a range similar to one of the previous studies on the human femoral artery at rest. Averaged over 15 cardiac cycles, $V_{\max }$ has previously been found to be $\sim 1.53$-fold higher than $V_{\text {mean }}$ [30]. By the use of a silicon tube to model the conduit artery, $V_{\max }$ have additionally been found to be approximately 1.75 -fold higher than $V_{\text {mean }}$ in vitro [31].

FBF at the cardiosystolic and cardiodiastolic phases during rhythmic muscle contractions. A linear relationship between leg blood flow and work intensity during DKE at $60 \mathrm{cpm}$ has previously been demonstrated with an ultrasound Doppler [1, 3, 12]. The present study also showed that FBF determined for both the cardiosystolic and cardiodiastolic phases increased linearly with work rate (Fig. 5). This supports a very close coupling between the metabolic activity and the blood pressure response during rhythmic muscle contractions of DKE. Furthermore, the increase in the mean and maximum $\mathrm{FBF}_{\text {exercise }} / \mathrm{FBF}_{\text {rest }}$ ratios were higher during the cardiodiastolic phase than during the cardiosystolic phase, though the increase in the exercising FBF ratio compared to rest was similar between the mean and maximum values during both the cardiosystolic 
and cardiodiastolic phases. A significant increase in both the mean and maximum exercising FBF values was also seen during the cardiodiastolic phase, but not during the cardiosystolic phase (Fig. 6).

Since the vessel walls are elastic, the area of the vessel lumen increases under increasing pressure. When the heart ejects its stroke volume into the aorta, the total volume of the aorta increases because of the increase in pressure. Therefore most of the ejected blood remains in the aorta (Windkessel model) during the cardiosystolic phase and flows to the periphery during the cardiodiastolic phase. The flow into the aorta when the heart contracts is composed of two terms. The first, called the compliant term, produces a volume expansion of the aorta itself. The other represents the outflow from the aorta to the periphery and is called the conductive term. Therefore during muscle contractions, the conductant blood flow is very important for supplying blood flow in the peripheral conduit artery to the contracting muscle with vasodilatation during the cardiodiastolic phase [8]. In previous studies, there is little emphasis in regard to the specific blood flow response during the cardiodiastolic phase. Thus its physiological impact may be to enhance exercise hyperemia during the cardiodiastolic phase of DKE. These present data suggest that the cardiodiastolic phase relative to the cardiosystolic phase obviously contributes more to the enhanced exerciseinduced hyperemia.

Conclusion. The major finding in the present study was an altered blood velocity profile in response to rhythmic muscle contractions at different exercise intensities. A less "steep" parabolic velocity profile was seen during the cardiodiastolic phase at lower muscle contraction intensities, whereas the "steepness" of the parabolic velocity profile increased during the cardiosystolic phase at higher muscle contraction intensities. Besides being influenced by the muscle contraction-relaxation-induced alterations in intramuscular pressure, the blood velocity profile and flow consequently appear to be modulated by the cardiac and blood pressure phases. Of major interest is that blood flow persists to be parabolic during both rest and exercise for both the cardiosystolic and cardiodiastolic phases, respectively. Consequently, blood flow remains laminar and parabolic, and there is no major turbulence that sustains to such an extent that the blood flow response is impaired. Instead the blood flow and the velocity profile are optimized to meet the metabolic requirements of the skeletal muscle under different conditions.
The authors wish to acknowledge the support given by Professor Bengt Saltin, the staff of The Copenhagen Muscle Research Centre, and Department of Preventive Medicine and Public Health at Tokyo Medical University, as well as the volunteers who participated in this study. T. Osada was a visiting research fellow from 1 January 2000 to 30 January 2002 at the CMRC. This study was supported by a grant from the Danish National Research Foundation (\#504-14) and by the Hippocratic Award at Tokyo Medical University in 2000 (T. Osada).

\section{REFERENCES}

1. Rådegran G: Ultrasound Doppler estimates of femoral artery blood flow during dynamic knee extensor exercise in humans. J Appl Physiol 83: 1383-1388, 1997

2. Rådegran G and Saltin B: Muscle blood flow at onset of dynamic exercise in humans. Am J Physiol Heart Circ Physiol 274: H314-H322, 1998

3. Osada T: Muscle contraction-induced limb blood flow variability during dynamic knee extensor. Med Sci Sports Exerc 36: 1149-1158, 2004

4. Hale JF, McDonald DA, and Womersley JR: Velocity profiles of oscillating arterial flow, with some calculations of viscous drag and the Reynolds numbers. J Physiol (Lond) 128: 629-640, 1955

5. Noordergraaf A: Circulatory System Dynamics, Academic Press, New York, 1978

6. Sheldon CD, Murie JA, and Quin RO: Ultrasonic Doppler spectral broadening in the diagnosis of internal carotid artery stenosis. Ultrasound Med Biol 9: 575-580, 1983

7. Burns PN and Jaffe CC: Quantitative flow measurements with Doppler ultrasound: techniques, accuracy, and limitations. Radiol Clin North Am 23: 641-657, 1985

8. Hatle L and Angelsen B: Doppler Ultrasound in Cardiology: Physical Principles and Clinical Applications, 2nd ed, Lea \& Febiger, Phyladelphia, Pennsylvania, 1985

9. Bishop JJ, Nance PR, Popel AS, Intaglietta M, and Johnson PC: Effect of erythrocyte aggregation on velocity profiles in venules. Am J Physiol Heart Circ Physiol 280: H222-H236, 2001

10. Andersen $P$ and Saltin B: Maximal perfusion of skeletal muscle in man. J Physiol (Lond) 366: 233-249, 1985

11. Andersen P, Adams RP, Sjøgaard G, Thorboe A, and Saltin B: Dynamic knee extension as model for study of isolated exercising muscle in humans. J Appl Physiol 59: 1647-1653, 1985

12. Osada T and Rådegran G: Femoral artery inflow in relation to external and total work rate at different knee extensor contraction rates. J Appl Physiol 92: 1325-1330, 2002

13. Sjøgaard G, Kiens B, Jørgensen K, and Saltin B: Intramuscular pressure, EMG and blood flow during lowlevel prolonged static contraction in man. Acta Physiol Scand 128: 475-484, 1986

14. Shoemaker JK, Hodge L, and Hughson RL: Cardiorespiratory kinetics and femoral artery blood velocity during dynamic knee extension exercise. J Appl Physiol 


\section{T. OSADA and G. RÅDEGRAN}

77: 2625-2632, 1994

15. Wesche J: The time course and magnitude of blood flow changes in the human quadriceps muscles following isometric contraction. J Physiol (Lond) 377: 445-462, 1986

16. Leyk D, EBfeld D, Baum K, and Stegemann J: Influence of calf muscle contractions on blood flow parameters measured in the arteria femoralis. Int J Sports Med 13: 588-593, 1992

17. Isnard R, Lechat $P$, Kalotka H, Chikr H, Fitoussi S, Salloum J, Golmard J-L, Thomas D, and Komajda M: Muscular blood flow response to submaximal leg exercise in normal subjects and in patients with heart failure. $J$ Appl Physiol 81: 2571-2579, 1996

18. Osada T, Katsumura T, Hamaoka T, Inoue S, Esaki K, Sakamoto A, Murase N, Kajiyama J, Shimomitsu T, and Iwane $\mathrm{H}$ : Reduced blood flow in abdominal viscera measured by Doppler ultrasound during one-legged knee extension. J Appl Physiol 86: 709-719, 1999

19. Osada T, Katsumura T, Hamaoka T, Murase N, Naka M, and Shimomitsu T: Quantitative effects of respiration on venous return during single knee extension-flexion. Int J Sports Med 23: 183-190, 2002

20. Gill RW: Measurement of blood flow by ultrasound: Accuracy and sources of error. Ultrasound Med Biol 11: 625-641, 1985

21. Whitmore RC: Rheology of the Circulation, Pergamon Press, Oxford, 1968

22. McDonald DA: The velocity profile in pulsatile flow. In: Blood Flow in Arteries, 2nd ed, Edward Arnold, London, pp 101-117, 1974

23. Sugawara M: Hemodynamics (Chapter 3). In: Handbook of Physiological Sciences, Cardiovascular Physi- ology, ed. Irisawa $\mathrm{H}$ and Kumada M, Igaku-Shoin Ltd. Tokyo, Vol 16, pp 198-216, 1991

24. Maeda N: Erythrocyte rheology in microcirculation. Jpn J Physiol 46: 1-14, 1996

25. Lunt MJ, Jenkinson DF, and Kerr D: Transcranial Doppler blood velocity measurement-The effect of changes in velocity profile. Ultrasound Med Biol 26: 1145-1151, 2000

26. Osada T, Katsumura T, Murase N, Sako T, Higuchi H, Kime R, Hamaoka T, and Shimomitsu T: Post-exercise hyperemia after ischemic and non-ischemic isometric handgrip exercise. J Physiol Anthropol Appl Human Sci 22: 299-309, 2003

27. Osada T: Exercise-related time course of pulsatility index in brachial artery following forearm exercise assessed by Doppler ultrasound. Tohoku J Exp Med 203: 241-252, 2004

28. Caro CG, Pedley TJ, Schroter RC, and Seed WA: The Mechanics of the Circulation, Oxford University Press, Oxford, 1978

29. Berne RM and Levy MN: Hemodynamics (Chapter 20), Cardiovascular system (Part IV). In: Principles of Physiology, ed. Berne RM and Levy MN, Mosby, A Times Mirror Company, pp 277-287, 1996

30. Holland CK, Brown JM, Scoutt LM, and Taylor KJ: Lower extremity volumetric arterial blood flow in normal subjects. Ultrasound Med Biol 24: 1079-1086, 1998

31. Moriyasu F, Ban N, Nishida O, Nakamura T, Miyake T, Uchino $\mathrm{H}$, Kanematsu $\mathrm{Y}$, and Koizumi S: Clinical application of an ultrasonic duplex system in the quantitative measurement of portal blood flow. J Clin Ultrasound 14: 579-588, 1986 